\title{
Prevalence of Methicillin-Resistant Staphylococcus aureus (MRSA) Among Patients Visiting the Emergency Room at a Tertiary Hospital in Brazil
}

\author{
Julival Ribeiro $^{1}$, John M. Boyce ${ }^{2}$ and Pedro Q. Zancanaro ${ }^{3}$
}

\author{
Hospital de Base ${ }^{1}$,Brasília, DF, Brazil; Miriam \\ Hospital ${ }^{2}$, Providence, RI, USA; University of Brasília ${ }^{3}$, \\ Brasília, DF, Brazil
}

\begin{abstract}
We surveyed patients seen at the emergency room at a tertiary hospital in Brazil from August to November 1997. All patients' $(n=600)$ anterior nares were cultured for MRSA; the results were confirmed by oxacillin disk diffusion methods and by detection of the mecA gene with PCR. Bacteria were found in $93.3 \%(560)$ of the patients and MRSA in $0.7 \%(n=4)$. One patient had community-acquired MRSA. We concluded that MRSA is uncommon among patients visiting the emergency room. The presence of community-acquired MRSA can lead to serious medical and epidemiological issues, although initial clinical presentation may not differ from that of infections with other staphylococci. The empirical use of vancomycin for suspected community-acquired infections is seldom warranted.

Key Words: MRSA, Staphylococcus aureus, emergency room, Brazil.
\end{abstract}

The first methicillin-resistant S. aureus (MRSA) strains were detected in Europe in 1960. During the following decades, MRSA became a leading cause of nosocomial infections worldwide and later became endemic in United States hospitals [1]. The National Nosocomial Infections Surveillance System (NNISS) recorded an increase of MRSA in large US hospitals, from $4 \%$ in the 1980 s to $50 \%$ in the late 1990 s [2]. Furthermore, the issue of multidrug resistance has made the care of these patients more difficult and expensive [3].

Cases of community-associated MRSA (CAMRSA) have been described throughout the world. These reports usually involved patients with recognized predisposing risk factors, such as intravenous drug use, serious illness (cardiovascular, diabetes mellitus, AIDS, carcinoma or renal failure), previous antibiotic therapy

Received on 29 July 2004; revised 22 November 2004. Address for correspondence: Dr. Julival F. Ribeiro. Comissão de Controle de Infecção Hospitalar - CCIH. Hospital de Base Address: SMHS Qd. 101 Brasília-DF, Brasil. Zip 70330-150. Email: jribeiro@abordo.com.br

The Brazilian Journal of Infectious Diseases 2005;9(1):52-55 (C) 2005 by The Brazilian Journal of Infectious Diseases and Contexto Publishing. All rights reserved. or residence in a nursing home [4-9]. Cases of community-acquired infections with methicillin-resistant S. aureus have also been reported [6,10-12]. Risk factors for infection with CA-MRSA and methicillinsusceptible S. aureus (MSSA) are comparable [8].

The emergence of CA-MRSA infections is of major concern for public health officials and clinicians. The first reported CA-MRSA infections occurred among Australian aboriginals and Native Americans in Canada in the early 1990s. The earliest reported cases of CAMRSA infection in the United States occurred in children with little or no recognized contact with hospitals or other healthcare institutions. Clusters of cases or outbreaks have occurred subsequently among children attending day-care centers, native Americans in Minnesota, North Dakota and Nebraska, athletic teams, prison inmates in Mississippi and Georgia, and among gay males in Los Angeles and San Francisco [13-15].

Healthcare-associated MRSA (HA-MRSA) strains continue to be a major problem in many institutions, and they now account for more than $50 \%$ of S. aureus recovered from patients in intensive care units (ICUs) and about $40 \%$ of the S. aureus isolated from nonICU patients. Risk factors for HA-MRSA include 
hemodialysis, illicit IV drug use, dermatitis, diabetes mellitus, burn unit patients, previous exposure to antimicrobial agents (especially cephalosporins, aminoglycosides and fluoroquinolones), prolonged hospitalization and severe underlying illness.

HA-MRSA-associated infections include surgical site infections, bacteremia and endocarditis, pneumonia, soft-tissue infections and urinary tract infections. The morbidity and mortality associated with HA-MRSA infections are at least equal to, and in some studies were greater than, those seen with MSSA infections. Most HA-MRSA strains are multi-drug resistant, leaving clinicians with few therapeutic alternatives [15].

We examined the prevalence of MRSA among patients seen at the emergency room/observation unit (ER) at a tertiary hospital in Brazil.

\section{Material and Methods}

We conducted a prospective survey from August to November 1997 to determine the prevalence of MRSA among patients admitted to the emergency room/observation unit at tertiary hospital in Brazil. Patients admitted from Monday toThursday and who remained in the internal medicine and surgical observation areas less than 48 hours were included. Patients' data was collected using a previously-prepared questionnaire. Medical residents used moistened swabs (Culturette-Becton-Dickinson Microbiology Systems) to collect material from patients' anterior nares. The swabs were inoculated directly onto blood agar plates and cultured in enrichment broth (fluid thioglycollate medium). All isolates of Staphylococcus were identified on the basis of colony characteristics, a positive catalase slide test and coagulase positivity (Slidex Staph-kit, bioMérieux). If no growth was observed on the plate or in the broth after 48 hours, it was considered negative. The material was replated from the broth when there was no growth in the initial plate. Antimicrobial susceptibility tests were performed using a Vitek machine with GPS-BS Gram-positive test panels. If MRSA was identified, additional confirmation tests using the oxacillin disk diffusion method (antimicrobial disks-Cecon-Brazil) were performed, according to NCCLS standards.

An agar screening method was used for the detection of methicillin-resistant $S$. aureus. The medium used was Mueller-Hinton agar supplemented with $4 \% \mathrm{NaCl}$ and $6 \mu \mathrm{g} / \mathrm{mL}$ oxacillin. Plates were inoculated with cotton swabs that had been dipped in a broth culture adjusted to the turbidity of a $0.5 \mathrm{McF}$ arland standard. After the inoculates had dried, the plates were incubated at $35^{\circ} \mathrm{C}$ for $24 \mathrm{hr}$; growth under these conditions was deemed indicative of isolation of MRSA. The isolates were also tested using BBL Crystal ID, according to the manufacturer's recommendation.

The MRSA isolates were also tested for the presence of mecA gene sequences using polymerase chain reaction (PCR) methods. PCR assays were performed by the Pathology Department, Miriam Hospital. The control strains included S. aureus ATCC 25923, ATCC 29213 and a known methicillin-resistant $S$. aureus strain.

\section{Results}

During the study period, 600 patients admitted to the emergency room were included. The median age was 38 years; $387(64.5 \%)$ were male and 213 were female $(35.5 \%)$. Two hundred and eight (34.7\%) patients had been admitted to a hospital within the last two years.

We were able to recover bacteria from 560 cultures (93.3\%). The most common isolates were coagulasenegative Staphylococci (366 isolates, 61\%), followed by methicillin-sensitive $S$. aureus (145 isolates, 24.3\%) and other isolates (45 isolates, 7.5\%). MRSA was only isolated in four patients $(0.7 \%)$. Three out of the four patients who had MRSA had been admitted to a hospital during the preceding two years, while the fourth had had no prior exposure to any healthcare facility.

\section{Discussion}

Methicillin-resistant Staphylococcus aureus may be nosocomial or community-acquired. From an 
epidemiological standpoint, MRSA can be defined as community-acquired if the positive culture was obtained outside hospital settings or within two days of hospital admission, and if the subject had no prior history of hospitalization within the preceding two years [16]. Boyce reviewed the incidence of community-acquired MRSA, and he pointed out that it is difficult to prove that the organism was not acquired as the result of a health care facility visit [17]. Other authors suggested that MRSA incidence is low in the community [1820]. Because MRSA colonization acquired in a hospital may persist for months to years, and given that most studies of community-acquired MRSA conducted in the 1990s did not exclude patients who had been hospitalized during the year preceding their MRSA infection, the frequency of true CA-MRSA has been a matter of considerable debate.

In spite of the high prevalence of MRSA in our hospital (35\%), we conclude that the prevalence of MRSA among patients visiting the emergency room has been very low $(0.7 \%)$ and that the empirical use of vancomycin for suspected community-acquired infections is seldom warranted. Only one patient had true CA-MRSA. This patient had no prior history of hospitalization for the previous two years. No risk factor was determined.

The presence of MRSA does not imply specific clinical manifestations. In fact, the clinical aspects of staphylococci infection correlate better with the site of acquisition (nosocomial or community-acquired) than with methicillin resistance. Although most affected individuals have presented with skin or soft tissue infections, some patients develop complications, such as fulminant pneumonia, bacteremia, endocarditis, or osteomyelitis. Fatal infections have occurred in otherwise healthy children [15].

Similarly, antimicrobial resistance is wider for nosocomial-acquired MRSA [11], and despite the presence of mecA gene in the community-acquired strains, additional resistance to quinolones, aminoglycosides, erythromycin, clindamycin and trimethoprim-sulfmethoxazole, mediated by transposons adjacent to the mec $\mathrm{A}$ gene, is rarely found, although resistance to these antimicrobials may be a matter of time. For these reasons, epidemiological surveys in the population may play a role in preventing the emergence of multi-drugresistant strains.

Recent methods are helping to determine the frequency of CA-MRSA. In addition to excluding patients who have been hospitalized during the previous year, isolates can be checked for: (1) resistance only to beta-lactams and erythromycin, (2) pulsed-field gel electrophoresis patterns that differ from local HAMRSA strains, and when possible, (3) the presence of staphylococcal cassette chromosome type IV. It is noteworthy that most of the true CA-MRSA strains that have these characteristics primarily cause skin and soft tissue infections (because many produce the Panton-Valentine leukocidin) or occasionally necrotizing pneumonia. [15]

Additional surveys of CA-MRSA may reveal the changes in Brazil during the last few years.

\section{References}

1. Oliveira C.D., Tomaz A., Lencastre H. Secrets of success of a human pathogen: molecular evolution of pandemic clones of methicillin-resistant Staphylococus aureus. The Lancet 2002;2(3):180-9.

2. Wenzel R.P., Nettleman M.D., Jones R.N., Pfaller M.A. Methicillin-resistant Staphylococus aureus: implications for the 1990s and effective control measures. Am J Med 1991;91:221S-7S.

3. Layton M.C., Hierholzer W., Paterson J.E. The Evolving Epidemiology of MRSA at a University Hospital. Infect Control Hosp Epidemiol 1995; 16:12-7.

4. Saravolatz L.D., Pohlod D.J., Arking L.M. Communityacquired MRSA infections: a new source of nosocomial outbreaks. Ann Intern Med 1982;97:325-9.

5. Saravolatz L.D., Markowitz N., Arking L., et al. MRSA. Epidemiological observations during a communityacquired outbreak. Ann Intern Med 1982;96:11-6.

6. Embil J., Remotar K., Romance L. et al. MRSA in tertiary care institutions on the Canada prairies 1990-1992. Infect Control Hosp Epidemiol 1994;15:644-51.

7. Mulligan M.E., Murray-Leisure K.A., Standiford H.C. et al. Methicillin-resistant S. aureus: a consensus review. Am J Med 1993;94:313-28.

8. Moreno F., Crisp C., Jorgensen J.H., Patterson J.E. Methicillin-resistant Staphylococcus aureus as a community organism. Clin lnfect Dis 1995;21:1308-12. 
9. Akram J., Glatt A.E. True Community-acquired methicillinresistant $S$. aureus bacteremia. Infect Control Hosp Epidemiol 1998; 19:106-7.

10. Haffman H., Davies H., Brett M. Current epidemiology of MRSA in New Zealand. Lablink 1997;4:25-6.

11. Maranan M.C., Moreira B., Boyle-Vavra S., Daum R.S. Antimicrobial resistance in staphylococci: epidemiology, molecular mechanism and clinical relevance. Infect Dis Clin North Am. 1997;11:813-49.

12. Kun-Chan W., et al. Characteristics of communityacquired methicillin-resistant Staphylococcus aureus in infants and children without known risk factors. Journal of Microbiology Immunology and Infection 2002;35(1):53-6.

13. Public Health Dispatch: Outbreaks of communityassociated methicillin-resistant Staphylococcus aureus skin infections - Los Angeles County, California, 2002-2003. MMWR 2003;52:88.

14. Herold B.C., Immergluck L.C., Maranan M.C., et al. Community-acquired methicillin-resistant Staphylococcus aureus in children with no identified predisposing risk. JAMA 1998;279:593-8.

15. Boyce J.M. Update on resistant Staphylococcus aureus infections. Clin Upd Infec Dis 2003; 6(2):1-4.

16. Salmenlina S., Lyytikinen O., Vuopio-Varkila J. Communityacquired methicillin-resistant Staphylococcus aureus, Finland. Emerg Infect Dis 2002; 8(6):602-7.

17. Boyce J.M., Landry M., Deetz T.R., DuPont H.L. Epidemiologic studies of an outbreak of nosocomial methicillin-resistant Staphylococcus aureus infections. Infect Control 1981;2:110-6.

18. Dan M., Moses Y., Poch F., et al. Carriage of methicillinresistant Staphylococcus aureus by non-hospitalized subjects in Israel. Infection 1992;6:30-3.

19. Abudu L., Blair I., Fraise A., Cheng K.K. Methicillinresistant Staphylococcus aureus (MRSA): a community-based prevalence survey. Epidemiol Infect 2001;126(3):351-6.

20. Boyce J.M. Are the epidemiology and microbiology of methicillin-resistant Staphylococcus aureus changing? JAMA 1998; 279:623-4. 